\title{
QED theory of the nuclear recoil effect in atoms
}

\author{
V.M. Shabaev \\ Department of Physics, St.Petersburg State University, \\ Oulianovskaya 1, Petrodvorets, St.Petersburg 198904, Russia
}

PACS number(s): 12.20.-m, 31.30.Jv, 31.30.Gs

\begin{abstract}
The quantum electrodynamic theory of the nuclear recoil effect in atoms to all orders in $\alpha Z$ is formulated. The nuclear recoil corrections for atoms with one and two electrons over closed shells are considered in detail. The problem of the composite nuclear structure in the theory of the nuclear recoil effect is discussed.
\end{abstract}




\section{Introduction}

The complete $\alpha Z$-dependence expressions for the nuclear recoil corrections to the energy levels of hydrogenlike atoms were first derived in [1]. These expressions consist of three contributions: the Coulomb contribution, the one-transverse-photon contribution, and the two-transverse-photon contribution. For a state $a$ the Coulomb contribution is given by (the relativistic units $\hbar=c=1$ are used in the paper)

$$
\begin{aligned}
\Delta E_{c} & =\Delta E_{c}^{(1)}+\Delta E_{c}^{(2)} \\
\Delta E_{c}^{(1)} & =\left\langle a\left|\frac{\mathbf{p}^{2}}{2 M}\right| a\right\rangle, \\
\Delta E_{c}^{(2)} & =\frac{2 \pi i}{M} \int_{-\infty}^{\infty} d \omega \delta_{+}^{2}(\omega)\left\langle a\left|\left[\mathbf{p}, V_{c}\right] G\left(\omega+\varepsilon_{a}\right)\left[\mathbf{p}, V_{c}\right]\right| a\right\rangle,
\end{aligned}
$$

where $|a\rangle$ is the unperturbed state of the Dirac electron in the Coulomb field of the nucleus, $V_{c}=-\frac{\alpha Z}{r}$ is the Coulomb potential of the nucleus, $\mathbf{p}$ is the momentum operator, $\delta_{+}(\omega)=\frac{i}{2 \pi}(\omega+i 0)^{-1}, G(\omega)=(\omega-H(1-i 0))^{-1}$ is the relativistic Coulomb Green function, $H=\boldsymbol{\alpha} \mathbf{p}+\beta m+V_{c}$. The onetransverse-photon contribution is

$$
\begin{aligned}
\Delta E_{\operatorname{tr}(1)}= & \Delta E_{\operatorname{tr}(1)}^{(1)}+\Delta E_{t r(1)}^{(2)}, \\
\Delta E_{t r(1)}^{(1)}= & -\frac{1}{2 M}\langle a|(\mathbf{D}(0) \mathbf{p}+\mathbf{p D}(0))| a\rangle, \\
\Delta E_{\operatorname{tr}(1)}^{(2)}= & -\frac{1}{M} \int_{-\infty}^{\infty} d \omega \delta_{+}(\omega)\langle a|\left(\left[\mathbf{p}, V_{c}\right] G\left(\omega+\varepsilon_{a}\right) \mathbf{D}(\omega)\right. \\
& \left.-\mathbf{D}(\omega) G\left(\omega+\varepsilon_{a}\right)\left[\mathbf{p}, V_{c}\right]\right)|a\rangle,
\end{aligned}
$$

where

$$
D_{m}(\omega)=-4 \pi \alpha Z \alpha_{l} D_{l m}(\omega),
$$

$\alpha_{l}(l=1,2,3)$ are the Dirac matrices, $D_{l m}(\omega)$ is the transverse part of the photon propagator in the Coulomb gauge. In the coordinate representation it is

$$
D_{i k}(\omega, \mathbf{r})=-\frac{1}{4 \pi}\left\{\frac{\exp (i|\omega| r)}{r} \delta_{i k}+\nabla_{i} \nabla_{k} \frac{(\exp (i|\omega| r)-1)}{\omega^{2} r}\right\} .
$$

The two-transverse-photon contribution is

$$
\Delta E_{t r(2)}=\frac{i}{2 \pi M} \int_{-\infty}^{\infty} d \omega\left\langle a\left|\mathbf{D}(\omega) G\left(\omega+\varepsilon_{a}\right) \mathbf{D}(\omega)\right| a\right\rangle .
$$


An attempt to derive the complete $\alpha Z$-dependence expressions for the nuclear recoil effect was previously undertaken in [2]. Except for the Coulomb contribution, the expressions found in [2] are in disagreement with the ones given above. A dominant part of this disagreement is caused by technical errors made in [2]. If we remove these errors from [2], a discrepancy remains in the one-transverse-photon contribution and, in addition, appears in the Coulomb contribution. This discrepancy was discussed in detail in [1].

Recently, the equations (1)-(5) were rederived in [3,4]. In Ref. [3], it was noted that the sum of these expressions can be written in the following compact form

$$
\Delta E_{t o t}=\frac{i}{2 \pi M} \int_{-\infty}^{\infty} d \omega\left\langle a\left|(\mathbf{p}-\mathbf{D}(\omega)) G\left(\omega+\varepsilon_{a}\right)(\mathbf{p}-\mathbf{D}(\omega))\right| a\right\rangle .
$$

The terms $\Delta E_{c}^{(1)}$ and $\Delta E_{t r(1)}^{(1)}$ can easily be calculated by using the virial relations for the Dirac equation [5]. Such a calculation gives [1]

$$
\Delta E^{(1)} \equiv \Delta E_{c}^{(1)}+\Delta E_{t r(1)}^{(1)}=\frac{m^{2}-\varepsilon_{a}^{2}}{2 M} .
$$

This simple formula contains all the nuclear recoil corrections within the $(\alpha Z)^{4} m^{2} / M$ approximation. The remaining terms (Eqs. (2),(4), and (5)) taken to the lowest order in $\alpha Z$ give the Salpeter corrections [6]. Evaluation of these terms to all orders in $\alpha Z$ in the range $Z=1-100$ was done in [7]. In particular, it was found in [7] that the complete (in $\alpha Z$ ) nuclear recoil correction, additional to the Salpeter one, to the Lamb shift $(n=2)$ in hydrogen constitutes $-1.32(6) \mathrm{kHz}$. This value almost coincides with the value of the $(\alpha Z)^{6} \mathrm{~m}^{2} / M$ correction found in [4,8-10].

The complete $\alpha Z$-dependence expressions for the nuclear recoil corrections for high $Z$ few-electron atoms were derived in [11]. These formulas were used in [7] to calculate the nuclear recoil corrections to all orders in $\alpha Z$ for high $Z$ lithiumlike atoms. As it follows from these formulas, within the $(\alpha Z)^{4} m^{2} / M$ approximation the nuclear recoil corrections can be obtained by averaging the operator

$$
H_{M}=\frac{1}{2 M} \sum_{s, s^{\prime}}\left(\mathbf{p}_{s} \cdot \mathbf{p}_{s^{\prime}}-\frac{\alpha Z}{r_{s}}\left(\boldsymbol{\alpha}_{s}+\frac{\left(\boldsymbol{\alpha}_{s} \cdot \mathbf{r}_{s}\right) \mathbf{r}_{s}}{r_{s}^{2}}\right) \cdot \mathbf{p}_{s^{\prime}}\right)
$$

with the Dirac wave functions. An independent derivation of this operator was done in [12]. The operator (8) was employed in [13] to calculate 
the $(\alpha Z)^{4} m^{2} / M$ corrections to the energy levels of two- and three-electron multicharged ions.

In the present paper we generalize the theory of the nuclear recoil effect to an arbitrary case of a many-electron atom. In particular, this generalization allows one to use as the zero approximation a potential that is different from the pure Coulomb field. In addition, it allows one to use the formalism in which the closed shells are refered to the vacuum state. In Sec. 2, we formulate the basic equations of the method. In Sec. 3 , we apply this method to an atom with one electron over closed shells. In Sec. 4, the case of an atom with two electrons over closed shells is considered. In Sec. 5, the problem of the composite nuclear structure is discussed.

\section{Basic formalism}

Like Refs. [3,4], we will consider the nucleus as a non-relativistic particle. In the Schrödinger representation and the Coulomb gauge, the Hamiltonian of the whole system is

$$
\begin{aligned}
H= & \int d \mathbf{x} \psi^{\dagger}(\mathbf{x})\left[\boldsymbol{\alpha} \cdot\left(-i \nabla_{\mathbf{x}}-e \mathbf{A}(\mathbf{x})\right)+\beta m\right] \psi(\mathbf{x}) \\
& +\frac{e^{2}}{8 \pi} \int d \mathbf{x} d \mathbf{y} \frac{\rho_{e}(\mathbf{x}) \rho_{e}(\mathbf{y})}{|\mathbf{x}-\mathbf{y}|}+\frac{1}{2} \int d \mathbf{x}\left(\mathcal{E}_{t}^{2}(\mathbf{x})+\mathcal{H}^{2}(\mathbf{x})\right) \\
& +\frac{e|e| Z}{4 \pi} \int d \mathbf{x} \frac{\rho_{e}(\mathbf{x})}{\left|\mathbf{x}-\mathbf{X}_{n}\right|}+\frac{1}{2 M}\left(\mathbf{P}_{n}-|e| Z \mathbf{A}\left(\mathbf{X}_{n}\right)\right)^{2} \\
& -\boldsymbol{\mu} \cdot \mathcal{H}\left(\mathbf{X}_{n}\right)
\end{aligned}
$$

where $m$ is the electron mass, $M$ is the nucleus mass, $e$ is the electron charge $(e<0), \mathbf{X}_{n}$ is the radius vector of the nucleus, $\mathbf{P}_{n}=-i \nabla_{\mathbf{X}_{n}}$, and $\boldsymbol{\mu}$ is the magnetic moment of the nucleus. The term $-\boldsymbol{\mu} \cdot \mathcal{H}$ causes the hyperfine splitting structure of atomic levels and will not be discussed here. The total momentum of the system is given by

$$
\mathbf{P}=\mathbf{P}_{n}+\mathbf{P}_{e}+\mathbf{P}_{f}
$$

where $\mathbf{P}_{e}=\int d \mathbf{x} \psi^{\dagger}(\mathbf{x})\left(-i \nabla_{\mathbf{x}}\right) \psi(\mathbf{x})$ is the electron-positron field momentum and $\mathbf{P}_{f}=\int d \mathbf{x}\left(\mathcal{E}_{t}(\mathbf{x}) \times \mathcal{H}(\mathbf{x})\right)$ is the electromagnetic field momentum. Since the total momentum is an integral of the motion we can restrict our consideration to the center-of-mass system $(\mathbf{P}=0)$ and, so, can express the nuclear 
momentum in terms of the electron-positron and electromagnetic field momenta

$$
\mathbf{P}_{n}=-\mathbf{P}_{e}-\mathbf{P}_{f}=-\int d \mathbf{x} \psi^{\dagger}(\mathbf{x})\left(-i \nabla_{\mathbf{x}}\right) \psi(\mathbf{x})-\int d \mathbf{x}\left(\mathcal{E}_{t}(\mathbf{x}) \times \mathcal{H}(\mathbf{x})\right) .
$$

Using this equation and the translation invariance we find

$$
\begin{aligned}
H= & \int d \mathbf{x} \psi^{\dagger}(\mathbf{x})\left[\boldsymbol{\alpha} \cdot\left(-i \nabla_{\mathbf{x}}-e \mathbf{A}(\mathbf{x})\right)+\beta m\right] \psi(\mathbf{x}) \\
& +\frac{e^{2}}{8 \pi} \int d \mathbf{x} d \mathbf{y} \frac{\rho_{e}(\mathbf{x}) \rho_{e}(\mathbf{y})}{|\mathbf{x}-\mathbf{y}|}+\frac{1}{2} \int d \mathbf{x}\left(\mathcal{E}_{t}^{2}(\mathbf{x})+\mathcal{H}^{2}(\mathbf{x})\right) \\
& +\frac{e|e| Z}{4 \pi} \int d \mathbf{x} \frac{\rho_{e}(\mathbf{x})}{|\mathbf{x}|}+\frac{1}{2 M}\left[-\int d \mathbf{x} \psi^{\dagger}(\mathbf{x})\left(-i \nabla_{\mathbf{x}}\right) \psi(\mathbf{x})\right. \\
& \left.-\int d \mathbf{x}\left(\mathcal{E}_{t}(\mathbf{x}) \times \mathcal{H}(\mathbf{x})\right)-|e| Z \mathbf{A}(0)\right]^{2} .
\end{aligned}
$$

Here we have omitted the hyperfine interaction term. The sum of the first four terms in (12) is the standard Hamiltonian of the electron-positron field interacting with the quantized electromagnetic field and with the classical Coulomb field of the nucleus $V_{c}=-\frac{\alpha Z}{r}$ ( a finite nuclear charge distribution can be taken into account by replacing $V_{c}$ with the potential of an extended nucleus (see Sec. 5)). The last term in (12) defines the nuclear recoil corrections of the first order in $m / M$. The part of this term containing the electromagnetic field momentum $\left(\mathbf{P}_{f}=\int d \mathbf{x}\left(\mathcal{E}_{t}(\mathbf{x}) \times \mathcal{H}(\mathbf{x})\right)\right)$ will contribute only in the first and higher orders in $\alpha$ and, so, will not be discussed here. It follows, to the zeroth order in $\alpha$ the nuclear recoil corrections can be calculated by adding to the standard Hamiltonian the following term

$$
\begin{aligned}
H_{M}= & \frac{1}{2 M} \int d \mathbf{x} \psi^{\dagger}(\mathbf{x})\left(-i \nabla_{\mathbf{x}}\right) \psi(\mathbf{x}) \int d \mathbf{y} \psi^{\dagger}(\mathbf{y})\left(-i \nabla_{\mathbf{y}}\right) \psi(\mathbf{y}) \\
& -\frac{e Z}{M} \int d \mathbf{x} \psi^{\dagger}(\mathbf{x})\left(-i \nabla_{\mathbf{x}}\right) \psi(\mathbf{x}) \mathbf{A}(0)+\frac{e^{2} Z^{2}}{2 M} \mathbf{A}^{2}(0)
\end{aligned}
$$

As is known, for description of an atomic system within QED it is convenient to use the interaction representation in the Furry picture. In such a theory the normal ordered form of $H_{M}$ taken in the interaction representation must be added to the interaction Hamiltonian. A simple way to derive formal 
expressions for the energy level shifts consists in using the technique developed in $[14,15]$. According to this technique, the energy shift $\Delta E_{a}=E_{a}-E_{a}^{(0)}$ of a single level $a$ of an $N$-electron atom is given by the formula

$$
\Delta E_{a}=\frac{\frac{1}{2 \pi i} \oint_{\Gamma} d E\left(E-E_{a}^{(0)}\right) \Delta g_{a}(E)}{1+\frac{1}{2 \pi i} \oint_{\Gamma} d E \Delta g_{a}(E)},
$$

where the contour $\Gamma$ surrounds only the unperturbed level $E=E_{a}^{(0)}$ (the contour is assumed to be traversed counterclockwise), $\Delta g_{a}(E)=g_{a}(E)-$ $g_{a}^{(0)}(E), g_{a}(E)$ is defined by

$$
\begin{aligned}
g_{a}(E) \delta\left(E-E^{\prime}\right)= & \frac{2 \pi}{i} \frac{1}{N !} \int_{-\infty}^{\infty} d p_{1}^{0} \ldots d p_{N}^{0} d p^{\prime 0}{ }_{1} \ldots d p^{\prime 0}{ }_{N} \\
& \times \delta\left(E-p_{1}^{0}-\ldots-p_{N}^{0}\right) \delta\left(E^{\prime}-p_{1}^{\prime 0}-\ldots-{p^{\prime}}_{N}\right) \\
& \times\left\langle u_{a}\left|G\left(p_{1}^{\prime 0}, \ldots, p^{\prime 0}{ }_{N} ; p_{1}^{0}, \ldots, p_{N}^{0}\right) \gamma_{1}^{0} \ldots \gamma_{N}^{0}\right| u_{a}\right\rangle
\end{aligned}
$$

with $G$ is the usual $N$-electron Green function

$$
\begin{aligned}
& G\left(p^{\prime 0}, \ldots, p^{\prime 0} ; p_{1}^{0}, \ldots, p_{N}^{0}\right) \\
& =(2 \pi)^{-2 N} \int_{-\infty}^{\infty} d x_{1}^{0} \ldots d x_{N}^{0} d x^{\prime 0}{ }_{1}^{0} \ldots d x^{\prime 0}{ }_{N} \\
& \times \exp \left(i p^{\prime 0}{ }_{1} x^{\prime 0}+\ldots+i p^{\prime 0}{ }_{N} x^{\prime 0}{ }_{N}-i p_{1}^{0} x_{1}^{0}-\ldots-i p_{N}^{0} x_{N}^{0}\right) \\
& \times\left\langle 0\left|T \psi\left(x_{1}^{\prime}\right) \ldots \psi\left(x_{N}^{\prime}\right) \bar{\psi}\left(x_{N}\right) \ldots \bar{\psi}\left(x_{1}\right)\right| 0\right\rangle,
\end{aligned}
$$

$\psi(x)$ is the electron-positron field operator in the Heisenberg representation, $\bar{\psi}(x)=\psi^{\dagger}(x) \gamma^{0}, u_{a}$ is the unperturbed atomic wave function, and $g_{a}^{(0)}(E)=$ $\left(E-E_{a}^{(0)}\right)^{-1}$ is the zeroth approximation of $g_{a}(E)$. In the first order of the perturbation theory we have

$$
\Delta E_{a}^{(1)}=\frac{1}{2 \pi i} \oint_{\Gamma} d E\left(E-E_{a}^{(0)}\right) \Delta g_{a}^{(1)}(E)
$$

The Green function $G$ is constructed using the Wick theorem after the transition in (16) to the interaction representation. The diagram technique rules for $G$ are considered in detail in [15]. Including $H_{M}$ in the interaction Hamiltonian gives the following additional lines and vertices to the diagram technique rules for $G$. 
1. Coulomb contribution.

An additional line ("Coulomb-recoil" line) appears to be

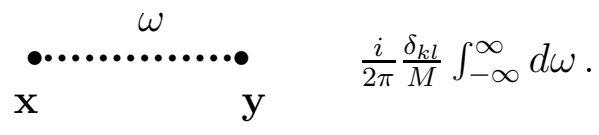

This line joins two vertices each of which corresponds to

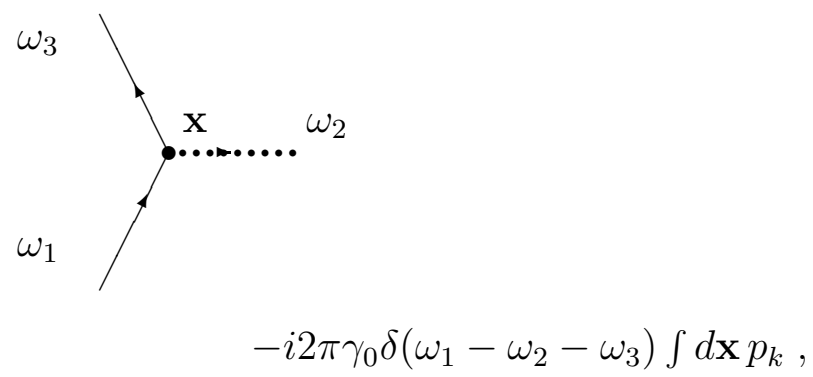

where $\mathbf{p}=-i \nabla_{\mathbf{x}}$ and $k=1,2,3$.

2. One-transverse-photon contribution.

An additional vertex on an electron line appears to be

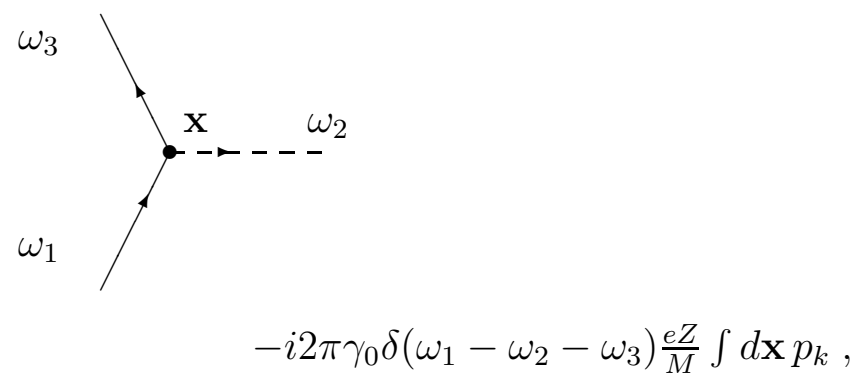

The transverse photon line attached to this vertex (at the point $\mathbf{x}$ ) is 


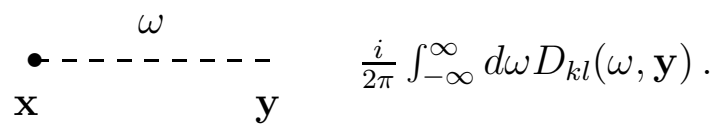

At the point $\mathbf{y}$ this line is to be attached to an usual vertex in which we have $-i e \gamma_{0} \alpha_{l} 2 \pi \delta\left(\omega_{1}-\omega_{2}-\omega_{3}\right) \int d \mathbf{y}\left(\right.$ see [15]), where $\alpha_{l}(l=1,2,3)$ are the usual Dirac matrices (we note here that in the notations of [15] $\alpha^{\mu}=(1, \boldsymbol{\alpha})$ and $\left.\alpha_{\mu}=(1,-\boldsymbol{\alpha})\right)$.

3. Two-transverse-photon contribution.

An additional line ("two-transverse-photon-recoil" line) appears to be

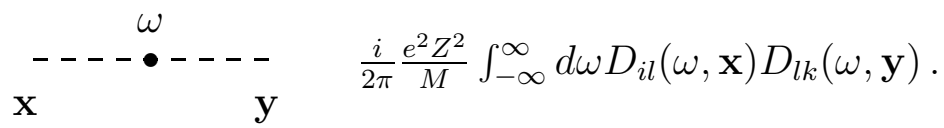

This line joins usual vertices (see the previous item).

An important advantage of the approach considered here, in comparison with one developed in $[1,11]$, consists in that the present method is suitable for arbitrary local potential $V(r)$ (e.g., a local version of the Hartree-Fock potential) used as the zero approximation. In addition, the transition to the formalism in which the role of the vacuum is played by closed shells can simply be realized by changing the sign of $i 0$ in the electron propagator denominators corresponding to the closed shells.

\section{One electron over closed shells}

Let us consider an atom with one electron over closed shells. In the zero approximation the electrons of the atom interact with the potential $V(r)$ which can be chosen to include approximately the electron-electron interaction. In the formalism with the closed shell states as well as the negative energy states refered to the vacuum, the electron propagator is given by

$$
S(\omega, \mathbf{x}, \mathbf{y})=\sum_{n} \frac{\psi_{n}(\mathbf{x}) \bar{\psi}_{n}(\mathbf{y})}{\omega-\varepsilon_{n}+i \eta_{n} 0}
$$

where $\eta_{n}=\varepsilon_{n}-\varepsilon_{F}$ and $\varepsilon_{F}$ is the Fermi energy which is chosen to be higher than the one-electron closed shell energies and lower than the energies of 
the one-electron states over the closed shells. In the simplest case of an one-electron atom $\eta_{n}=\varepsilon_{n}$.

To find the Coulomb nuclear recoil correction we have to calculate the contribution of the diagram shown in Fig. 1. According to the diagrams technique rules given in the previous section and [15] we obtain

$$
\Delta g_{a}^{(1)}=\frac{1}{\left(E-E_{a}^{(0)}\right)^{2}} \frac{1}{M} \frac{i}{2 \pi} \int_{-\infty}^{\infty} d \omega \sum_{n} \frac{\left\langle a\left|p_{i}\right| n\right\rangle\left\langle n\left|p_{i}\right| a\right\rangle}{\omega-\varepsilon_{n}+i \eta_{n} 0} .
$$

The formula (17) gives

$$
\Delta E_{c}=\frac{1}{M} \frac{i}{2 \pi} \int_{-\infty}^{\infty} d \omega \sum_{n} \frac{\left\langle a\left|p_{i}\right| n\right\rangle\left\langle n\left|p_{i}\right| a\right\rangle}{\omega-\varepsilon_{n}+i \eta_{n} 0} .
$$

Using the identities

$$
\begin{aligned}
& \frac{1}{x+i 0}=\frac{\pi}{i} \delta(x)+\mathrm{P} \frac{1}{x} \\
& \frac{1}{x-i 0}=\pi i \delta(x)+\mathrm{P} \frac{1}{x}
\end{aligned}
$$

one can get

$$
\begin{aligned}
\Delta E_{c} & =\frac{1}{2 M} \sum_{n} \frac{\eta_{n}}{\left|\eta_{n}\right|}|\langle a|\mathbf{p}| n\rangle|^{2} \\
& =\frac{1}{2 M}\left\langle a\left|\mathbf{p}^{2}\right| a\right\rangle-\frac{1}{M} \sum_{\varepsilon_{n}<\varepsilon_{F}}|\langle a|\mathbf{p}| n\rangle|^{2} .
\end{aligned}
$$

The one-transverse-photon nuclear recoil correction corresponds to the diagrams shown in Fig. 2. A similar calculation gives

$$
\begin{aligned}
\Delta E_{t r(1)}= & \frac{4 \pi \alpha Z}{M} \frac{i}{2 \pi} \int_{-\infty}^{\infty} d \omega \sum_{n}\left\{\frac{\left\langle a\left|p_{i}\right| n\right\rangle\left\langle n\left|\alpha_{k} D_{i k}\left(\varepsilon_{a}-\omega\right)\right| a\right\rangle}{\omega-\varepsilon_{n}+i \eta_{n} 0}\right. \\
& \left.+\frac{\left\langle a\left|\alpha_{k} D_{i k}\left(\varepsilon_{a}-\omega\right)\right| n\right\rangle\left\langle n\left|p_{i}\right| a\right\rangle}{\omega-\varepsilon_{n}+i \eta_{n} 0}\right\} .
\end{aligned}
$$

By using the identity

$$
\frac{1}{\omega-\varepsilon_{n}+i \eta_{n} 0}=\frac{1}{\omega-\varepsilon_{a}+i 0}+\frac{\varepsilon_{n}-\varepsilon_{a}}{\left(\omega-\varepsilon_{a}+i 0\right)\left(\omega-\varepsilon_{n}+i \eta_{n} 0\right)}
$$


and the equation (21), the expression (24) can easily be transformed to the following

$$
\begin{aligned}
\Delta E_{\operatorname{tr}(1)}= & \Delta E_{\operatorname{tr}(1)}^{(1)}+\Delta E_{\operatorname{tr}(1)}^{(2)}, \\
\Delta E_{\operatorname{tr}(1)}^{(1)}= & \frac{4 \pi \alpha Z}{2 M}\left\langle a\left|\left(p_{i} \alpha_{k} D_{i k}(0)+\alpha_{k} D_{i k}(0) p_{i}\right)\right| a\right\rangle \\
\Delta E_{\operatorname{tr}(1)}^{(2)}= & \frac{4 \pi \alpha Z}{M} \int_{-\infty}^{\infty} d \omega \delta_{+}\left(\omega-\varepsilon_{a}\right) \sum_{n}\left\{\frac{\left\langle a\left|\left[p_{i}, V\right]\right| n\right\rangle\left\langle n\left|\alpha_{k} D_{i k}\left(\varepsilon_{a}-\omega\right)\right| a\right\rangle}{\omega-\varepsilon_{n}+i \eta_{n} 0}\right. \\
& \left.-\frac{\left\langle a\left|\alpha_{k} D_{i k}\left(\varepsilon_{a}-\omega\right)\right| n\right\rangle\left\langle n\left|\left[p_{i}, V\right]\right| a\right\rangle}{\omega-\varepsilon_{n}+i \eta_{n} 0}\right\}
\end{aligned}
$$

The two-transverse-photon nuclear recoil correction is defined by the diagram shown in Fig. 3. We find

$$
\begin{aligned}
\Delta E_{t r(2)}= & \frac{(4 \pi \alpha Z)^{2}}{M} \frac{i}{2 \pi} \int_{-\infty}^{\infty} d \omega \sum_{n} \\
& \times \frac{\left\langle a\left|\alpha_{i} D_{i l}\left(\varepsilon_{a}-\omega\right)\right| n\right\rangle\left\langle n\left|\alpha_{k} D_{l k}\left(\varepsilon_{a}-\omega\right)\right| a\right\rangle}{\omega-\varepsilon_{n}+i \eta_{n} 0} .
\end{aligned}
$$

As it follows from the equations (20), (24), and (27), the sum of all the contributions can be written in the following compact form

$$
\begin{aligned}
\Delta E_{t o t}= & \frac{1}{M} \frac{i}{2 \pi} \int_{-\infty}^{\infty} d \omega\langle a|\left(p_{i}+4 \pi \alpha Z \alpha_{l} D_{l i}(\omega)\right) \\
& \times G\left(\omega+\varepsilon_{a}\right)\left(p_{i}+4 \pi \alpha Z \alpha_{m} D_{m i}(\omega)\right)|a\rangle,
\end{aligned}
$$

where $G(\varepsilon)=\sum_{n} \frac{|n\rangle\langle n|}{\varepsilon-\varepsilon_{n}+i \eta_{n} 0}$ is the electron Green function.

In the case of a hydrogenlike atom, the expressions derived here coincide with ones given in Sec. 1.

\section{Two electrons over closed shells}

Consider now an atom with two electrons over closed shells (a general case of $N$ electrons over closed shells can be considered in the same way). For simplicity, we take as the unperturbed wave function the one-determinant wave function

$$
u=\frac{1}{\sqrt{2}} \sum_{P}(-1)^{P} \psi_{P a}\left(\mathbf{x}_{\mathbf{1}}\right) \psi_{P b}\left(\mathbf{x}_{\mathbf{2}}\right) .
$$


The nuclear recoil correction is the sum of the one-electron and two-electron contributions. Using the diagram technique rules from [15] and the Sec. 2 and the formula (17) one easily finds that the one-electron contribution is equal to the sum of the expressions (28) for $a$ and $b$ states. The twoelectron contributions correspond to the diagrams shown in Fig. 4-6. The two-electron Coulomb contribution is

$$
\begin{aligned}
\Delta E_{c}^{(i n t)}= & \frac{1}{M} \frac{1}{2 \pi i} \oint_{\Gamma} d E\left(E-E^{(0)}\right)\left\{\left(\frac{i}{2 \pi}\right)^{2} \int_{-\infty}^{\infty} d p^{0} d p^{0}\right. \\
& \times \sum_{P}(-1)^{P} \frac{1}{p^{0}-\varepsilon_{P a}+i 0} \frac{1}{E-p^{0}-\varepsilon_{P b}+i 0} \\
& \times \frac{1}{p^{0}-\varepsilon_{a}+i 0} \frac{1}{E-p^{0}-\varepsilon_{b}+i 0} \\
& \left.\times\left\langle P a\left|p_{i}\right| a\right\rangle\left\langle P b\left|p_{i}\right| b\right\rangle\right\} .
\end{aligned}
$$

Integrating over $p^{0}, p^{\prime 0}$, and $E$ we get

$$
\Delta E_{c}^{(i n t)}=\frac{1}{M} \sum_{P}(-1)^{P}\left\langle P a\left|p_{i}\right| a\right\rangle\left\langle P b\left|p_{i}\right| b\right\rangle .
$$

A similar calculation of the one-transverse-photon contribution gives

$$
\begin{aligned}
\Delta E_{t r(1)}^{(i n t)}= & \frac{4 \pi \alpha Z}{M} \sum_{P}(-1)^{P}\left[\left\langle P a\left|p_{i}\right| a\right\rangle\left\langle P b\left|\alpha_{k} D_{k i}\left(\varepsilon_{P b}-\varepsilon_{b}\right)\right| b\right\rangle\right. \\
& \left.+\left\langle P a\left|\alpha_{k} D_{k i}\left(\varepsilon_{P a}-\varepsilon_{a}\right)\right| a\right\rangle\left\langle P b\left|p_{i}\right| b\right\rangle\right] .
\end{aligned}
$$

Finally, for the two-transverse-photon contribution we find

$$
\begin{aligned}
\Delta E_{t r(2)}^{(i n t)}= & \frac{(4 \pi \alpha Z)^{2}}{M} \sum_{P}(-1)^{P}\left[\left\langle P a\left|\alpha_{k} D_{k i}\left(\varepsilon_{P a}-\varepsilon_{a}\right)\right| a\right\rangle\right. \\
& \times\left\langle P b\left|\alpha_{m} D_{m i}\left(\varepsilon_{P b}-\varepsilon_{b}\right)\right| b\right\rangle .
\end{aligned}
$$

The sum of the two-electron contributions (31)-(33) can be written in the following compact form

$$
\begin{aligned}
\Delta E_{\text {tot }}^{(i n t)}= & \frac{1}{M} \sum_{P}(-1)^{P}\left\langle P a\left|p_{i}+4 \pi \alpha Z \alpha_{l} D_{l i}\left(\varepsilon_{P a}-\varepsilon_{a}\right)\right| a\right\rangle \\
& \times\left\langle P b\left|p_{i}+4 \pi \alpha Z \alpha_{m} D_{m i}\left(\varepsilon_{P b}-\varepsilon_{b}\right)\right| b\right\rangle .
\end{aligned}
$$


The formulas (31)-(34) coincide with the related expressions found for high- $Z$ few-electron atoms in [11] (see also [7]). The difference is only the present expressions (31)-(34) are not restricted to the case of the pure Coulomb zero approximation.

\section{Composite nuclear structure}

The problem of the composite nuclear structure in the nuclear recoil theory was first discussed by Salpeter [6]. In Ref. [6], it was shown that the calculations based on the assumption that the nucleus is a point Dirac particle of electric charge $|e| Z$ and mass $M$ are valid for composite nuclei (independently of the nuclear spin), if the distance between the nuclear levels is large compared with the distance between the atomic (electrons + field) levels contributing to the nuclear recoil effect. In this section we consider how this result can be derived within the approach developed in the present paper.

Let us assume, for simplicity, that the nucleus is a bound state of a twoparticle system (e.g., a core with a mass $m_{1}$ and a charge $e_{1}$ and a valent nucleon with a mass $m_{2}$ and a charge $e_{2}$ ). In this case the sum of the last three terms in equation (9) must be replaced by $H_{1}+H_{2}+H_{3}$, where

$$
\begin{aligned}
H_{1}= & \frac{e}{4 \pi} \int d \mathbf{x} \rho_{e}(\mathbf{x})\left(\frac{e_{1}}{\left|\mathbf{x}-\mathbf{x}_{1}\right|}+\frac{e_{2}}{\left|\mathbf{x}-\mathbf{x}_{2}\right|}\right) \\
H_{2}= & \frac{1}{2 m_{1}}\left(\mathbf{p}_{1}-e_{1} \mathbf{A}\left(\mathbf{x}_{1}\right)\right)^{2}+\frac{1}{2 m_{2}}\left(\mathbf{p}_{2}-e_{2} \mathbf{A}\left(\mathbf{x}_{2}\right)\right)^{2} \\
& +U\left(\mathbf{x}_{1}-\mathbf{x}_{2}\right), \\
H_{3}= & -\boldsymbol{\mu}_{s}^{(1)} \cdot \mathcal{H}\left(\mathbf{x}_{1}\right)-\boldsymbol{\mu}_{s}^{(2)} \cdot \mathcal{H}\left(\mathbf{x}_{2}\right) .
\end{aligned}
$$

Here $U\left(\mathbf{x}_{1}-\mathbf{x}_{2}\right)$ describes the interaction between the nuclear particles (for simplicity, we assume that $U$ does not depend of the spins) and $\boldsymbol{\mu}_{s}^{(1)}$ and $\boldsymbol{\mu}_{s}^{(2)}$ are the intristic magnetic moments of the nuclear particles. Introducing the center-of-nucleus-mass variables

$$
\mathbf{X}_{n}=\frac{m_{1} \mathbf{x}_{1}+m_{2} \mathbf{x}_{2}}{m_{1}+m_{2}}, \quad \mathbf{x}_{n}=\mathbf{x}_{1}-\mathbf{x}_{2}
$$

we have

$$
\mathbf{p}_{1}=\frac{m_{1}}{m_{1}+m_{2}} \mathbf{P}_{n}+\mathbf{p}_{n}, \quad \mathbf{p}_{2}=\frac{m_{2}}{m_{1}+m_{2}} \mathbf{P}_{n}-\mathbf{p}_{n},
$$


where $\mathbf{P}_{n}=-\nabla_{\mathbf{x}_{n}}$ and $\mathbf{p}_{n}=-\nabla_{\mathbf{x}_{n}}$. As in the Sec. 2, we can restrict our consideration to the center-of-atom-mass system $\left(\mathbf{P}=\mathbf{P}_{n}+\mathbf{P}_{e}+\mathbf{P}_{f}=0\right)$. So, the total nuclear momentum $\mathbf{P}_{n}$ is given by the equation (11). In terms of the variables $\mathbf{X}_{n}$ and $\mathbf{x}_{n}$ the operator $H_{2}$ can be represented in the form

$$
H_{2}=H_{\mu}+H_{M}
$$

where

$$
H_{\mu}=\frac{\mathbf{p}_{n}^{2}}{2 \mu}+U\left(\mathbf{x}_{n}\right)
$$

and $\mu=\left(m_{1} m_{2}\right) /\left(m_{1}+m_{2}\right)$. The Hamiltonian $H_{\mu}$ describes the intristic states of the nucleus. Let us denote the wave function of the nuclear state which is under the consideration by $\phi_{a}\left(\mathbf{x}_{n}\right)$. The wave function of the whole system in the zero approximation is the product of $\phi_{a}\left(\mathbf{x}_{n}\right)$ and the atomic wave function calculated using the operator $\left\langle\phi_{a}\left|H_{1}\right| \phi_{a}\right\rangle$ as the interaction with the nucleus (we assume here and subsequently that the distance between the nuclear energy levels is large compared with the distance between the atomic levels). The operator $\left\langle\phi_{a}\left|H_{1}\right| \phi_{a}\right\rangle$ describes the interaction of electrons with the extended nucleus charge. The $m / M$ corrections are calculated by perturbation theory. Using the fact that the nuclear size $\left(\sim\left|\mathbf{x}_{n}\right|\right)$ is much smaller than the atomic size, we expand the vector $\mathbf{A}$ in powers of $\mathbf{x}_{n}$. Taking into account that $\phi_{a}\left(\mathbf{x}_{n}\right)$ is of a definite parity we find to the lowest orders

$$
\begin{aligned}
& \left\langle\phi_{a}\left|H_{M}\right| \phi_{a}\right\rangle \\
& =\left\langle\phi_{a}\right|\left\{\frac{\mathbf{P}_{n}^{2}}{2 M}-\frac{\left(e_{1}+e_{2}\right)}{2 M}\left(\mathbf{P}_{n} \cdot \mathbf{A}\left(\mathbf{X}_{n}\right)+\mathbf{A}\left(\mathbf{X}_{n}\right) \cdot \mathbf{P}_{n}\right)\right. \\
& \quad+\left(\frac{e_{1}^{2}}{2 m_{1}}+\frac{e_{2}^{2}}{2 m_{2}}\right) \mathbf{A}^{2}\left(\mathbf{X}_{n}\right)-\frac{1}{2 M}\left(\frac{e_{1} m_{2}}{m_{1}}+\frac{e_{2} m_{1}}{m_{2}}\right) \\
& \left.\times\left[\mathbf{p}_{n}\left(\mathbf{x}_{n} \cdot \nabla_{\mathbf{X}_{n}}\right) \mathbf{A}\left(\mathbf{X}_{n}\right)+\left(\mathbf{x}_{n} \cdot \nabla_{\mathbf{X}_{n}}\right) \mathbf{A}\left(\mathbf{X}_{n}\right) \mathbf{p}_{n}\right]\right\}\left|\phi_{a}\right\rangle .
\end{aligned}
$$

The last term in (42) can be transformed to

$$
\begin{aligned}
-\frac{1}{2 M}\left(\frac{e_{1} m_{2}}{m_{1}}\right. & \left.+\frac{e_{2} m_{1}}{m_{2}}\right) \int d \mathbf{x}_{n} \phi_{a}^{*}\left(\mathbf{x}_{n}\right)\left(\mathbf{x}_{n} \times \mathbf{p}_{n}\right) \phi_{a}\left(\mathbf{x}_{n}\right) \mathcal{H}\left(\mathbf{X}_{n}\right) \\
& =-\left(\frac{e_{1}}{2 m_{1}}\left\langle\mathbf{l}_{1}\right\rangle+\frac{e_{2}}{2 m_{2}}\left\langle\mathbf{l}_{2}\right\rangle\right) \mathcal{H}\left(\mathbf{X}_{n}\right)
\end{aligned}
$$


where $\mathbf{l}_{1}$ and $\mathbf{l}_{2}$ are the orbital moments of the nuclear particles in the centerof-nucleus-mass system. Adding this term to the term $H_{3}$ gives the total operator of the hyperfine interaction $-\boldsymbol{\mu} \cdot \mathcal{H}\left(\mathbf{X}_{n}\right)$, where $\boldsymbol{\mu}=\boldsymbol{\mu}_{l}^{(1)}+\boldsymbol{\mu}_{s}^{(1)}+$ $\boldsymbol{\mu}_{l}^{(2)}+\boldsymbol{\mu}_{s}^{(2)}$ is the total magnetic moment of the nucleus and $\boldsymbol{\mu}_{l}^{(i)}=\frac{e_{i}}{2 m_{i}} \mathbf{l}_{i}$. Due to the operator $H_{M}$ contains the term

$$
H_{M}^{\prime}=-\left(\frac{e_{1}}{m_{1}}-\frac{e_{2}}{m_{2}}\right) \mathbf{p}_{n} \cdot \mathbf{A}\left(\mathbf{X}_{n}\right),
$$

there is a contribution of the order $m / M$ from the second order of the perturbation theory. For a state $a$ of the whole system we have

$$
\Delta E_{a}^{\prime}=\left(\frac{e_{1}}{m_{1}}-\frac{e_{2}}{m_{2}}\right)^{2} \sum_{n \neq a} \frac{\left\langle a\left|\mathbf{p}_{n} \cdot \mathbf{A}\left(\mathbf{X}_{n}\right)\right| n\right\rangle\left\langle n\left|\mathbf{p}_{n} \cdot \mathbf{A}\left(\mathbf{X}_{n}\right)\right| a\right\rangle}{E_{a}-E_{n}} .
$$

Assuming that the energy difference between the nuclear state which is under the consideration and the other nuclear states contributing to the sum in the equation (45) is large compared with the corresponding energy differences between the atomic (electrons + field) states which give a dominant contribution to $\Delta E_{a}^{\prime}$, we replace $E_{a}-E_{n}$ in Eq. (45) with $\epsilon_{a}-\epsilon_{n}$, where $\epsilon_{a}$ and $\epsilon_{n}$ are the nuclear energies. Using the identity $\mathbf{p}_{n}=i \mu\left[H_{\mu}, \mathbf{x}_{n}\right]$ we find

$$
\begin{aligned}
\Delta E_{a}^{\prime}= & \left(\frac{e_{1}}{m_{1}}-\frac{e_{2}}{m_{2}}\right)^{2}\left\langle\Phi_{a}\left|\mathbf{A}^{2}\left(\mathbf{X}_{n}\right)\right| \Phi_{a}\right\rangle \frac{i}{2} \mu \sum_{n \neq a} \frac{1}{\epsilon_{a}-\epsilon_{n}} \\
& \times\left[\left\langle\phi_{a}\left|\left[H_{\mu}, \mathbf{x}_{n}\right]\right| \phi_{n}\right\rangle\left\langle\phi_{n}\left|\mathbf{p}_{n}\right| \phi_{a}\right\rangle+\left\langle\phi_{a}\left|\mathbf{p}_{n}\right| \phi_{n}\right\rangle\left\langle\phi_{n}\left|\left[H_{\mu}, \mathbf{x}_{n}\right]\right| \phi_{a}\right\rangle\right] \\
& =\left(\frac{e_{1}}{m_{1}}-\frac{e_{2}}{m_{2}}\right)^{2}\left\langle\Phi_{a}\left|\mathbf{A}^{2}\left(\mathbf{X}_{n}\right)\right| \Phi_{a}\right\rangle \frac{i}{2} \mu \sum_{n \neq a} \\
& \times\left[\left\langle\phi_{a}\left|\mathbf{x}_{n}\right| \phi_{n}\right\rangle\left\langle\phi_{n}\left|\mathbf{p}_{n}\right| \phi_{a}\right\rangle-\left\langle\phi_{a}\left|\mathbf{p}_{n}\right| \phi_{n}\right\rangle\left\langle\phi_{n}\left|\mathbf{x}_{n}\right| \phi_{a}\right\rangle\right] \\
= & \left(\frac{e_{1}}{m_{1}}-\frac{e_{2}}{m_{2}}\right)^{2}\left\langle\Phi_{a}\left|\mathbf{A}^{2}\left(\mathbf{X}_{n}\right)\right| \Phi_{a}\right\rangle \frac{i}{2} \mu\left\langle\phi_{a}\left|\left[\mathbf{x}_{n}, \mathbf{p}_{n}\right]\right| \phi_{a}\right\rangle \\
& =-\frac{1}{2}\left(\frac{e_{1}}{m_{1}}-\frac{e_{2}}{m_{2}}\right)^{2} \mu\left\langle\Phi_{a}\left|\mathbf{A}^{2}\left(\mathbf{X}_{n}\right)\right| \Phi_{a}\right\rangle,
\end{aligned}
$$

where $\left|\phi_{a}\right\rangle$ is the nuclear wave function and $\left|\Phi_{a}\right\rangle$ is the atomic (electrons + field) wave function. Combaining this term with the equation (42) we find that the nuclear recoil correction of the first order in $m / M$ is defined by the operator

$$
H_{M}=\frac{\mathbf{P}_{n}^{2}}{2 M}-\frac{\left(e_{1}+e_{2}\right)}{2 M}\left(\mathbf{P}_{n} \cdot \mathbf{A}\left(\mathbf{X}_{n}\right)+\mathbf{A}\left(\mathbf{X}_{n}\right) \cdot \mathbf{P}_{n}\right)
$$




$$
\begin{aligned}
& +\frac{\left(e_{1}+e_{2}\right)^{2}}{2 M} \mathbf{A}^{2}\left(\mathbf{X}_{n}\right) \\
& =\frac{1}{2 M}\left(\mathbf{P}_{n}-|e| Z \mathbf{A}\left(\mathbf{X}_{n}\right)\right)^{2}
\end{aligned}
$$

So, within the approximations made in the derivation of this result, the effects of the composite nuclear structure can be neglected.

\section{Acknowledgements}

Valuable conversations with I.P.Grant, S.G.Karshenboim, I.B.Khriplovich, G.Soff, D.A.Tel'nov, and A.S.Yelkhovsky are gratefully acknowledged. The research described in this publication was made possible in part by Grant No. 95-02-05571a from RFBR. 


\section{References}

[1] V.M.Shabaev, Teor.Mat.Fiz. 63, 394 (1985) (Theor.Math.Phys. 63, 588 (1985)); In: Papers at First Soviet-British Symposium on Spectroscopy of Multicharged Ions (Academy of Sciences, Troitsk, 1986), pp. 238-240.

[2] M.A.Braun, Zh.Eksp.Teor.Fiz. 64, 413 (1973) (Sov. Phys. JETP 37, 211 (1973)).

[3] A.S.Yelkhovsky, Preprint BINP 94-27 (Budker Inst. of Nuclear Physics, Novosibirsk, 1994); hep-th/9403095 (1994); Zh.Eksp.Teor.Fiz. 110, 431 (1996) (JETP 83, 230 (1996)).

[4] K.Pachucki and H.Grotch, Phys.Rev.A 51, 1854 (1995).

[5] J.Epstein and S.Epstein, Am.J.Phys. 30, 266 (1962); V.M.Shabaev, Vestn.Leningrad.Univ. N4, 15 (1984); J.Phys.B 24, 4479 (1991).

[6] E.E.Salpeter, Phys.Rev. 87, 328 (1952); H.A.Bethe and E.E.Salpeter, Quantum Mechanics of One- and Two-Electron Atoms (Springer, Berlin, 1957).

[7] A.N.Artemyev, V.M.Shabaev, and V.A.Yerokhin, Phys.Rev. A 52, 1884 (1995); J.Phys.B 28, 5201 (1995).

[8] I.B.Khriplovich, A.I.Milstein and A.S.Yelkhovsky, Phys.Scr. T 46, 252 (1993).

[9] R.N.Fell, I.B.Khriplovich, A.I.Milstein and A.S.Yelkhovsky, Phys.Lett.A 181, 172 (1993).

[10] E.A.Golosov, I.B.Khriplovich, A.I.Milstein, and A.S.Yelkhovsky, Zh.Eksp.Teor.Fiz. 107, 393 (1995) (JETP 80, 208 (1995)).

[11] V.M.Shabaev, Yad.Fiz. 47, 107 (1988) (Sov.J.Nucl.Phys. 4769 (1988)).

[12] C.W.Palmer, J.Phys.B 20, 5987 (1987).

[13] V.M.Shabaev and A.N.Artemyev, J.Phys.B 27, 1307 (1994). 
[14] V.M.Shabaev, In: Many-particles Effects in Atoms, ed. U.I.Safronova, p. 15 (AN SSSR, Nauchnyi Sovet po Spektroskopii, 1988) (in Russian); Izv.Vuz.Fiz. 33, 43 (1990) (Sov.Phys.J. 33, 660 (1990)).

[15] V.M.Shabaev and I.G.Fokeeva, Phys.Rev.A 49, 4489 (1994); V.M.Shabaev, Phys.Rev.A 50, 4521 (1994). 


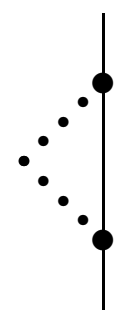

Figure 1: Coulomb nuclear recoil diagram.

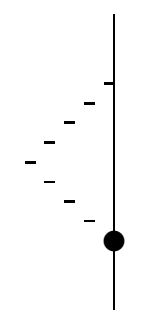

a

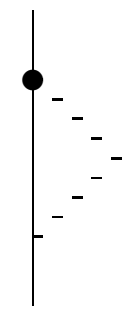

b

Figure 2: One-transverse-photon nuclear recoil diagrams. 


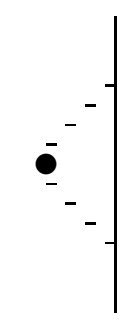

Figure 3: Two-transverse-photon nuclear recoil diagram.

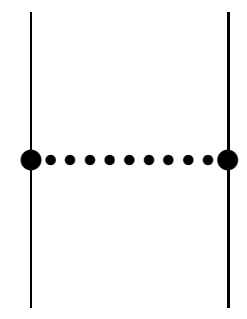

Figure 4: Two-electron Coulomb nuclear recoil diagram. 


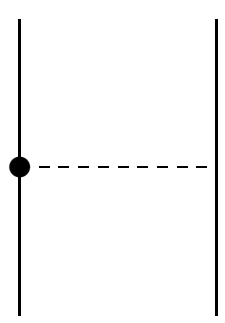

a

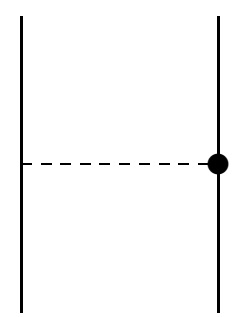

$\mathrm{b}$

Figure 5: Two-electron one-transverse-photon nuclear recoil diagrams.

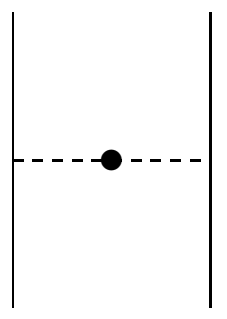

Figure 6: Two-electron two-transverse-photon nuclear recoil diagram. 